edly, a matter of judgement where the limit of the dead band should be set.

In connection with negative ABGs, it should be recalled that the commonly adopted rules for masking in $\mathrm{BC}$ tests lead to a bias towards positive ABGs. For the more accurate measurement of $\mathrm{BC}$ thresholds required for medicolegal purposes, masking should always be used, thereby achieving consistency with the test conditions in the experimental studies on which national and international calibration standards are based. Then as many negative $A B G s$ as positive ones will appear in sensorineural cases: indeed, there would be something wrong with the calibration standards if they did not

Turning to the statistical argument, we would go along with our critics' statement that 'it is not correct to assert that a particular confidence limit based on this dispersion [the inter-subject dispersion of $A B G$ values] represents uncertainty in the air-bone gap'. We should more correctly have written about confidence limits on the deviation of a measured $\mathrm{ABG}$ from the (normal) population mean. From this point of view, the position that an individual occupies in the distribution of all individuals is indistinguishable from a random variate. With regard to the extra allowance for random errors of measurement, it is a fine point whether this should be added in a linear as opposed to a root-mean-square manner. We might well have set the dead-band limit at three standard deviations and ignored the possible measurement error, and still arrived at the same value of $15 \mathrm{~dB}$. The fact is, as already stated, that the particular number selected is a matter of judgement related to the 'balance of probabilities' between the two hypotheses above. In practice, we do not feel that there is much leeway either side of $15 \mathrm{~dB}$ if one is to avoid, on the one hand, an excessive burden of proof and, on the other hand, falling into the trap of the reductio ad absurdum: $20 \mathrm{~dB}$ is, surely, evidence enough to justify the assumption that a real conductive loss is present, whilst $10 \mathrm{~dB}$ is still well within the normal inter-subject range and defies any firm conclusion.

Finally, our critics point out that uncertainties in the audiometric expression of impairment should be handled in the domain of disability. We agree, and that principle applies not only to the uncertainty attaching to the true cochlear impairment, but equally to the airconduction HTLs. However, as a practical matter, it turns out to be very difficult to write an explicit formula for the percent disability equivalent to so many decibels of uncertainty in ABG. This is not a difficulty of principle, but arises simply because the formula entails two cumbersome non-linear transformations: ABG to conductive threshold shift (Carhart's effect) and hearing threshold level to percent disability, respectively. The algebraic complication can, of course, quite easily be outflanked in any given case, by evaluating the disability percentage for two values of $A B G$ separated by their estimated decibel uncertainty. For example, one could insert the measured $A B G$ and the measured value minus $15 \mathrm{~dB}$ and see how the difference between the resulting estimates of disability comes out. In effect, this is close to our own way of dealing with the problem, except that we recommend a built-in margin and taking the single output value as the most appropriate measure.

All the complications of $\mathrm{BC}$ audiometry would evap- orate if only someone would invent and perfect a way of directly measuring the acoustical input to the cochlea. Yours faithfully,

R. R. A. Coles, M. E. Lutman and D. W. Robinson

\section{The feeding pharyngostomy}

Dear Sir,

We read with keen interest the article 'The feeding pharyngostomy: an alternative approach to enteral feeding' by D. G. John and C. P. Fielder (Journal of Laryngology and Otology, 1991; 105, 451-453).

During the last five years we have done over 150 pharyngostomies to feed patients with upper aerodigestive tract cancer in the post-operative period and in advanced stages of the disease. Our technique, though basically similar to the one described, has some differences: 1. In some patients with advanced cancer, it is not possible to pass a feeding tube down, due to obstruction by the tumour. So we first attempt to pass the tube down into the stomach through the mouth, and only if it succeeds do we make an incision in the neck to bring out the proximal end of the tube through the wound in the neck. This avoids unnecessary trauma to the neck of patients in whom a feeding tube cannot be passed down the oesophagus into the stomach. An alternate method of feeding is employed in such cases.

2. The feeding tube is brought out of the skin at the anterior border of the sternomastoid muscle near the angle of the mandible. The proximal end of the tube is caught between the jaws of a Mixter dissecting forceps which is directed laterally and superiorly behind the posterior tonsillar pillar. An incision is made at the point where the forceps tents up the skin, and the proximal end of the tube is brought outside. As the track through which the tube traverses the neck is inclined from the pharyngeal mucosa to the skin, dependent drainage does not occur through the track. So the chance of a persistent cervical fistula after removal of the tube is very low. In fact we have not had even one case of persistent leak from the neck after removal of the tube, till now.

Yours faithfully,

Paul Sebastian, M.S., Thomas Cherian, M.S., M.Ch., Assistant Profesor, Associate Professor,

Division of Surgical Oncology,

Regional Cancer Centre,

Trivandrum-695 011,

India.

\section{Reply}

Dear Sir,

I was most interested in the comments of Profs. Sebastian and Cherian concerning their large series of pharyngostomies.

I would agree with their first point, that in patients where oesophageal or post-cricoid obstruction is a possibility a nasogastric tube must be passed first. Since writing the paper I have performed two pharyngostomies for neuromuscular disease, under local anaesthesia and in these cases it is also absolutely necessary to pass the tube before making any external incision.

In their second point it is suggested that the tube is 
brought out near the angle of mandible rather than below the tip of the hyoid bone to avoid salivary leak. As stated in our paper, two of 33 cases required closure of a persistent saliva leak (both simply performed under local anaesthesia). Also, one patient out of six recent cases developed referred otalgia requiring removal of the pharyngostomy tube (and replacement by gastrostomy). I will certainly try the alternate technique suggested by Sebastian and Cherian.
Patient comfort and ease of changing of long-term feeding tubes are also important. I will be interested to see whether patients are more aware of a tube entering behind the tonsil than in the pyriform fossa and whether it is cosmetically acceptable for feeding at home.

Yours faithfully,

Colin Fielder, F.R.C.S.,

Consultant ENT Surgeon, Swansea. 